\title{
Hemisection as an Alternative Treatment for Decayed Multirooted Abutment: A Case Report
}

\author{
Dr. Hrushikesh Nirmal ${ }^{1}$, Dr. Saurabh Chaturvedi ${ }^{2}$, Dr.Mudita Chaturvedi ${ }^{3}$, \\ Dr.Tanuja Deshpande ${ }^{4}$ \\ 1- Senior Lecturer, Department of Prosthodontics, College of dental sciences, Rau, Indore, Madhya \\ Pradesh, India \\ 2- Senior Lecturer, Department of Prosthodontics, Career Post graduate institute of dental sciences \& \\ Hospital, Lucknow, Uttar Prades, India \\ 3- Senior Lecturer, Department of Oral and Maxillofacial Pathology, Career Post graduate institute of \\ dental sciences \& Hospital, Lucknow, Uttar Prades,India \\ 4- Senior Lecturer, Department of Prosthodontics, SDM College of Dental Sciences, Dharwad, India
}

\begin{abstract}
Conservation of tooth structure has always been the prime aim of the restorative procedures. Hemisection is a conservative treatment approach for multirooted teeth affected with caries, bone loss and furcation involvement. Proper case selection is of utmost importance for successful treatment. This case report presents a review on hemisection or premolarization and describes the management of mandibular molar with furcation involvement via hemisection.
\end{abstract}

Keywords - Furcation, Hemisection, Premolarization.

\section{Introduction}

Today's dentistry is based on conservation. Aim of any treatment modality is to preserve the natural, but proper periodontic, prosthodontic and endodontic assessment for appropriate selection of cases is important ${ }^{1}$.

Root resection is the process by which one or more of the roots of a tooth are removed at the level of the furcation while leaving the crown and remaining roots in function. ${ }^{2}$ Farrar $^{3}$ introduced this root-resection procedure, which has been used to treat Class II and III furcation-involved molars. Through root-resection therapy, furcation-involved molars can be converted to non-furcated single-root teeth and provide a favorable environment for oral hygiene maintenance.

The prognosis of root resection has been well-documented. Some investigators ${ }^{4,5}$ reported that rootresected molars had more than $90 \%$ survival rate, whereas other investigators ${ }^{6-9}$ reported that $\sim 30 \%$ of resected molars failed over a 10-year period. Some investigators compared the prognosis of root-resection therapy to that of implant therapy. Kinsel et al. ${ }^{10}$ reviewed the result of root-resection therapy and single implants in molar regions. They reported a $15.9 \%$ failure rate for root-resection therapy, whereas single implants showed a $3.6 \%$ failure rate. They stated that root-resection therapy had poor long-term results unless a high level of expertise was available in all applicable disciplines. In contrast, Fugazzotto ${ }^{11}$ reported 15 -year cumulative success rates of 96.8\% for root- resected molars and $97.0 \%$ for molar implants. He concluded that molar root- resection therapy and implant therapy had a high degree of functional success. The most important factors for the prognosis of root-resection therapy have been suggested. ${ }^{4-9,12,13}$ The following should be considered when deciding which root should be retained: the amount of supporting tissue around the roots, the root and root canal anatomy in relation to the endodontic treatment, the periapical condition, and the mobility of each separated root. ${ }^{13}$ Molars with minimal supporting bone or deep osseous craters in the furcation area are poor candidates for root-resection therapy as a result of the recurrent periodontal breakdown of resected molars. ${ }^{6}$ However, there are few reports showing the factors that actually influence the survival rate of resected molars. ${ }^{9}$

Misch ${ }^{14}$ stated that of all available options for replacing single posterior teeth-a removable prosthesis, a resin-bonded fixed partial denture, three-unit fixed restorations, maintenance of the posterior space or endosseous implants - the single-tooth implant generally is the best choice. The advantages of the single posterior endosseous implant are improved hygiene, decreased risk of dental caries, maintenance of bone and improved prosthesis longevity, and success rates as high as $97 \%{ }^{14}$

Indications for root resection treatment ${ }^{15}$ -

\section{Periodontal indications}

- $\quad$ severe bone loss affecting one or more roots untreatable with regenerative procedures

- class II or III furcation invasions or involvements 
- severe recession or dehiscence of a root

Endodontic indications

- inability to successfully treat and fill a canal

- root fracture or root perforation

- severe root resorption

- root decay

Prosthetic indications

- $\quad$ severe root proximity inadequate for a proper embrasure space

- root trunk fracture or decay with invasion of the

- biological width

\title{
Contraindications to root resection treatment
}

\author{
General contraindications to periodontal surgery \\ - Systemic factors \\ - Poor oral hygiene \\ Factors associated with local anatomy \\ - Fused roots \\ - Unfavorable tissue architecture \\ Endodontic factors \\ - Retained roots endodontically untreatable \\ - Excessive endodontic instrumentation of retained roots \\ - Excessive deepening of pulp chamber floor \\ Restorative factors \\ - Internal root decay \\ - Presence of a cemented post in the remaining root \\ Strategic considerations ${ }^{12}$. \\ - Consider adjacent teeth available for conventional prosthetic restoration \\ - Consider removable prosthesis \\ - Consider implants
}

\section{Case Report}

A 52 year old male patient reported to Department of Prosthodontics for crowning of root canal treated 36. Clinical evaluation of same tooth revealed grade II furcation involvement, periodontal pocket of $4 \mathrm{~mm}$ depth on distal aspect (Fig.1) and root extension of caries. Radiographic evaluation of the same tooth revealed severe bone loss on the distal aspect and radiolucency in the furcation area (Fig.2). There was a three unit fixed partial denture replacing missing 46. It was decided to extract the distal root owing to presence of bone loss associated with it, root extension of caries, and to eliminate the furcation allowing production of maintainable architecture on the mesial root.

During the surgical procedure crevicular incision was given from 34 to 37 (Fig.3). Full thickness mucoperiosteal flap was reflected. The mesial and distal roots were sectioned at the level of the furcation using long tapered fissure diamond. The distal root was extracted (Fig.4) and the socket was irrigated adequately with normal saline to remove bony chips. The flap was replaced and simple interrupted sutures were placed. A finishing diamond bur was used to smoothen the distal surface of the mesial root and its coronal portion. The occlusal table was reduced in size to redirect the forces along the long axis of the mesial root. The surgical site was covered with a periodontal dressing (coe-pack TM GC America INC.ALSIP, IL, U.S.A) (Fig.5). Four weeks following surgery, complete healing in the surgical site was observed.

In the prosthodontic phase of therapy metal fixed partial denture involving mandibular second molar and the retained mesial half of mandibular first molar was planned. Mesial half of mandibular first molar was selected as abutment as radiographic evaluation revealed adequate bone support (Fig.6). The edentulous space between mandibular second molar and the retained mesial half of mandibular first molar measured $6 \mathrm{~mm}$ mesiodistally and $9 \mathrm{~mm}$ cervico- occlusally. 37 and mesial root of 36 were prepared with supragingival chamfer finish (Fig.7) line to receive metal retainer. Putty-light body impression was made using polyvinyl siloxane impression material. Provisional restoration was fabricated using indirect technique and was cemented in place.

To enhance the oral hygiene maintenance modified sanitary pontic design was selected. All metal bridge was fabricated subsequently and cemented in place (Fig.8). 


\section{Figures}

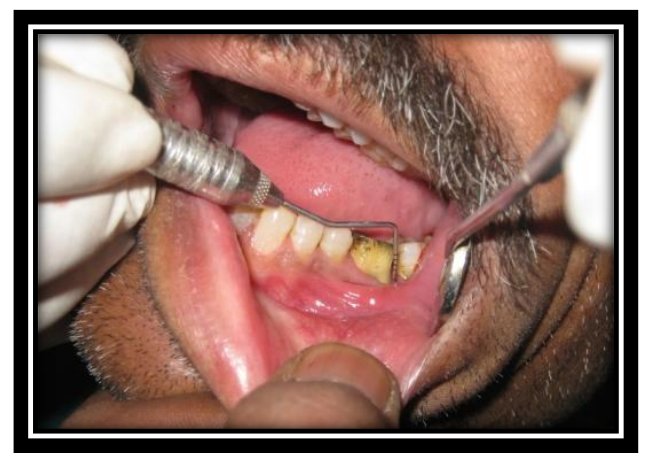

Fig.1- Periodontal pocket of $4 \mathrm{~mm}$ depth on distal aspect

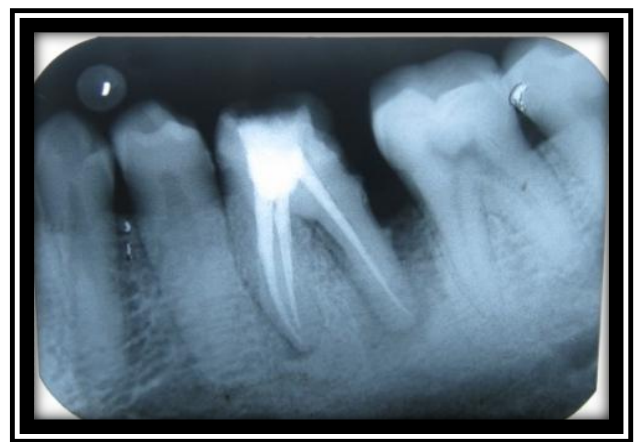

Fig.2- Bone loss on the distal aspect and radiolucency in the furcation area

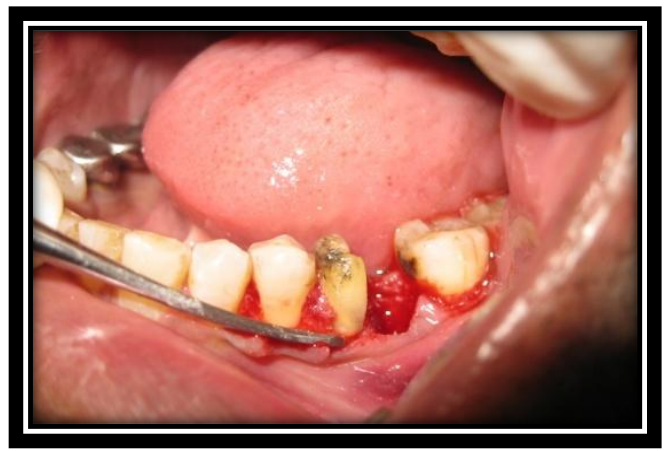

Fig. 3 - Crevicular incision from 34 to 37 with extraction site .

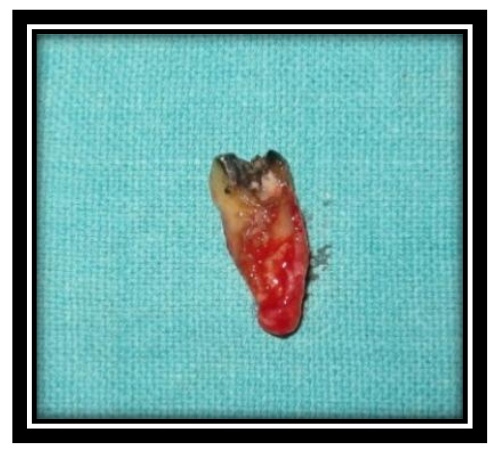

Fig.4-Extracted distal root 


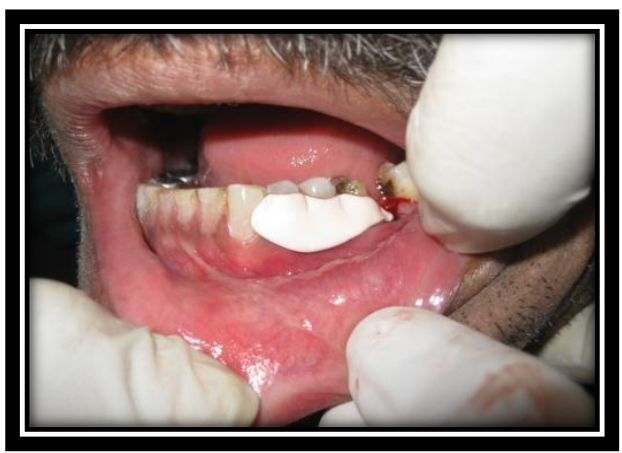

Fig.5- The surgical site covered with a periodontal dressing .

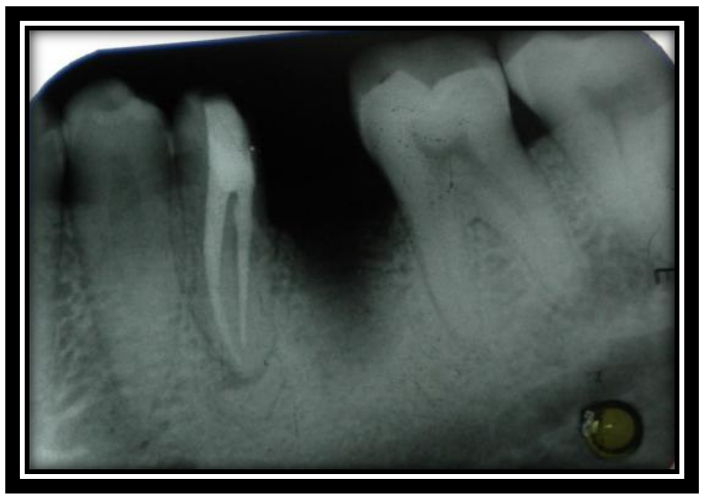

Fig.6- Mesial half of mandibular first molar with adequate bone support

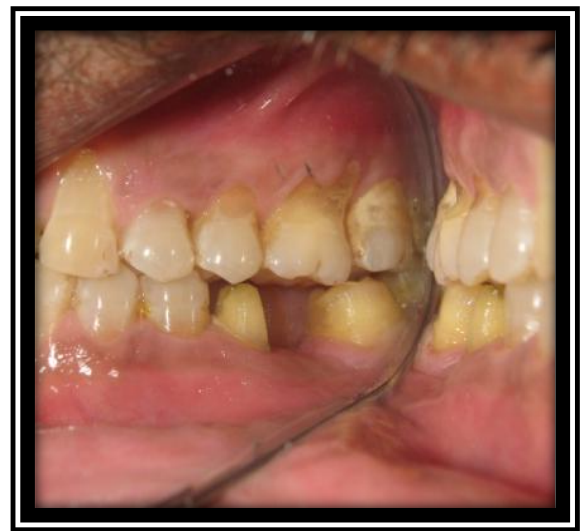

Fig.7- Prepared abutments with supragingival chamfer finish line to receive metal retainer.

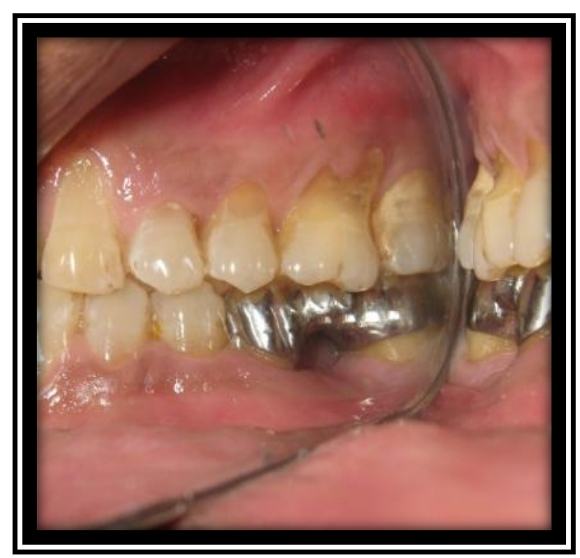

Fig.8- All metal bridge cemented in place . 


\section{Discussion}

For this patient, hemisection was selected as treatment of root decay and bone loss with the abutment of a fixed prosthesis. Implant therapy was considered but not chosen because of financial considerations; instead, a 3-unit fixed partial denture, extending from the hemisected first molar to the second molar, was completed. The distal root was resected because of the location of the decay and bone loss. The literature on distal root resection is limited; more often, this root is retained and the mesial root is removed. However, the distal root is broader and straighter, making it more suitable as an abutment. The distal aspect of mesial root is usually heavily fluted. The curvature and fluting may increase the potential for root perforation during endodontic therapy or complicate post placement. These anatomic features may also result in increased incidence of vertical root fracture.

The smaller size of the occlusal tables, under-contouring of the embrasure spaces and ensuring that the crown margin encompasses the furcation are all factors in the high success rates observed with hemisection therapy.

\section{CONCLUSION}

Root resection can successfully treat specific furcation defects that cannot be solved by other surgical and nonsurgical approaches. Complications with these resective procedures are not rare but are usually avoidable when specific endodontic, surgical and restorative guidelines are followed. Hemisection has received acceptance as a conservative and dependable dental treatment and teeth so treated have endured the demands of function.

\section{References}

[1]. DeSanctis M, Murphy KG. The role of resective periodontal surgery in the treatment of furcation defects. Periodontol 2000 2000;22:154-168

[2]. American Academy of Periodontology. Glossary of Periodontal Terms. Chicago: American Academy of Periodontology; 2001:45

[3]. Farrar JN. Radical and heroic treatment of alveolar abscess by amputation of roots of teeth. Dental Cosmos 1884;26:79.

[4]. Carnevale G, Di Febo G, Tonelli MP, Marin C, Fuzzi M. A retrospective analysis of the periodontal-prosthetic treatment of molars with interradicular lesions. Int J Periodontics Restorative Dent 1991:11:189-205.

[5]. Carnevale G, Pontoriero R, di Febo G. Long-term effects of root-resective therapy in furcation-involved molars. A 10-year longitudinal study. J Clin Periodontol 1998;25:209-214.

[6]. Langer B, Stein SD, Wagenberg B. An evaluation of root resections. A ten-year study. J Periodontol 1981; 52:719-722.

[7]. Buhler H. Evaluation of root-resected teeth. Results after 10 years. J Periodontol 1988;59:805-810.

[8]. Green EN. Hemisection and root amputation. J Am Dent Assoc 1986;112:511-518.

[9]. Blomlof L, Jansson L, Appelgren R, Ehnevid H, Lindskog S. Prognosis and mortality of root-resected molars. Int J Periodontics Restorative Dent 1997; 17: 190-201.

[10]. Kinsel RP, Lamb RE, Ho D. The treatment dilemma of the furcated molar: Root resection versus single-tooth implant restoration. A literature review. Int J Oral Maxillofac Implants 1998;13:322-332.

[11]. Fugazzotto PA. A comparison of the success of root resected molars and molar position implants in function in a private practice: Results of up to 15-plus years. J Periodontol 2001;72:1113-1123.

[12]. Minsk L, Poison AM. The role of root resection in the age of dental implants. Compend Contin Educ Dent 2006;27:384-388.

[13]. Hamp SE, Nyman S, Lindhe J. Periodontal treatment of multirooted teeth. Results after 5 years. J Clin Periodontol 1975;2:126-135.

[14]. Misch CE. Endosteal implants for posterior single tooth replacement:alternatives, indications, contraindications, and limitations. J Oral Implantol 1999;25:80-94

[15]. Basaraba N. Root amputation and tooth hemisection. Dent Clin North Am 1969;13:121-132. 